\title{
Neonatal Sepsis is Still a Major Problem in Tertiary Care Hospitals
}

\author{
Rina Das ${ }^{1}$, Sabina Yeasmin², Purushuttam Kumar³, Indrani Bhattacharyya ${ }^{4}$ \\ ${ }^{1}$ Department of Microbiology, CNMC, Kolkata, West Bengal, India. ${ }^{2}$ Department of Microbiology, \\ CNMC, Kolkata, West Bengal, India. ${ }^{3}$ Department of Microbiology, CNMC, Kolkata, West Bengal, India. \\ ${ }^{4}$ Department of Microbiology, CNMC, Kolkata, West Bengal, India.
}

\section{ABSTRACT}

\section{BACKGROUND}

In a developing country like India, one of the most important health hazards is neonatal sepsis. The ever full SNCUs of the hospitals are living proof of this. The neonates both early and late are extremely vulnerable to all organisms, both in the community and much more so in the hospital. Neonatal sepsis contributes to infant mortality rate in a very significant way. Nosocomial neonatal sepsis is much more dangerous and contributes to morbidity and mortality in a very big way.

\section{METHODS}

In this study, neonatal sepsis was assessed by culture of blood, pus from infected umbilical stump, and CSF sample received from SNCU. The isolated organisms were identified phenotypically following our laboratory protocol. Antibiotic sensitivity testing was done by Kirby-Bauer method, following CLSI guidelines.

\section{RESULTS}

It was found that $48 \%$ of the total samples received from SNCU were growth positive. Klebsiella pneumoniae was isolated in maximum number of cases, $42.85 \%$ followed by Staphylococcus aureus $18.53 \%$. Antibiotic sensitivity test by KirbyBauer method showed that all Gram-positive cocci were sensitive to vancomycin. There was only one linezolid resistant Staphylococcus aureus. Among the Gramnegative bacilli $100 \%$ isolates were sensitive to carbapenems. Acinetobacter baumannii and Pseudomonas aeruginosa isolates were multidrug resistant.

\section{CONCLUSIONS}

SNCU admitted babies are extremely vulnerable to septicaemia. The ever-changing drug resistant patterns of the infecting organisms is a perineal problem. Thus, infection control measures are to be strictly enforced in this setting.

\section{KEY WORDS}

Gram Positive Bacteria, Gram Negative Bacteria, Septicaemia, Kirby-Bauer Method, Resistant, SNCU
Corresponding Author: Dr. Indrani Bhattacharyya, Flat No. B4, 37J/1D, Raja Manindra Road, Kolkata-700037, West Bengal, India. E-mail: indranichaudhuri@yahoo.com

DOI: $10.14260 / \mathrm{jemds} / 2020 / 131$

Financial or Other Competing Interests: None.

How to Cite This Article:

Das R, Yeasmin S, Kumar P, et al. Neonatal sepsis is still a major problem in tertiary care hospitals. J. Evolution Med. Dent. Sci. 2020;9(09):588-592, DOI: 10.14260/jemds/2020/131

Submission 18-10-2019, Peer Review 03-02-2020, Acceptance 11-02-2020, Published 02-03-2020. \section{(1)}




\section{BACKGROUND}

India is a developing country bogged down by a huge population, poverty, illiteracy, lack of health education and ignorance about hygiene. All these factors lead to an unusual load on the health sector which is already highly compromised due to insufficient financial support and huge disparity between patient number and health workers. Most infections and many non - infectious diseases are found in large numbers, both in urban and rural areas. A scientific study of infectious diseases in our country shows that persons of extreme age groups are more vulnerable than young adults and adults possibly because the former group has a low immune status which may be due to various reasons.1,2 SNCUs have the most vulnerable group of patients in the hospital. The sick neonates admitted here do not have a properly developed immune system. Maternal causes such as anaemia, malnutrition, and certain diseases like diabetes, hypertension etc contribute to the immune deficiency in the newborn. There is still a large population of pregnant mothers who have no access to antenatal care. The necessary vaccines are not available to them. Even today, in remote areas domiciliary child delivery is still quite common. All these factors contribute to make neonatal septicaemia a huge burden in India.

Neonatal sepsis is a clinical syndrome characterized by systemic signs of infection accompanied by bacteraemia or septicaemia, in the first month of life. If sepsis occurs within 72 hours of birth, the condition is known as Early Neonatal Sepsis and if it occurs later but within 4 weeks, the condition is called Late Neonatal Sepsis. As newborn babies do not have a well-developed immune system, they are extremely susceptible to all varieties of infection particularly those caused by bacteria. This vulnerability among neonates is further aggravated by lack of proper hygiene, explosive population, economic constraints, old wife's tales like applying cow dung to the umbilical stump etc. Lack of spacing between child births does not allow the new mother to look after the newborn as she has to fend for one or more older children. Communication from remote villages to the nearest health facility is often very scarce and cannot be afforded by the family. Hence, remote areas are even today almost inaccessible to health facilities. However, it has been noted that timely hospitalization, appropriate treatment and proper nursing care reduces the morbidity and mortality to a large extent. This study has been performed to find out the percentages of different bacteria isolated from neonatal sepsis cases within a time span of one year, along with their antibiotic sensitivity patterns, in a postgraduate medical institute in Kolkata.

\section{Objectives}

1. To identify the common bacterial pathogens among neonates admitted in SNCU (sick neonatal care unit) of Calcutta National Medical College Hospital.

2. To find out the drug sensitivity pattern of the organisms isolated in order to prepare a streamlined antibiotic policy specifically meant for SNCU patients.

\section{METHODS}

This is a cross-sectional observational study conducted at Calcutta National Medical College and Hospital, Kolkata from May 2017 to April 2018. All specimens sent from SNCU for microbiological examination within the above time period were included in the study.

\section{Clinical Material}

1. Blood- As per departmental protocol for each blood sample a blood culture bottle was requisitioned. For SNCU patients, blood culture medium consisted of a monophasic brain heart infusion broth, $10 \mathrm{ml}$ in volume supplied in McCartney's bottle. The blood collected directly in the bottle was sent to the Microbiology department and incubated immediately at $37^{\circ} \mathrm{C}$.

2. Pus- Swabs were sent from umbilical stumps.

3. CSF- In case of sick neonates, CSF was collected either by cisternal puncture or lumbar puncture and CSF was sent to the laboratory in sterile test tube.

A total number of 532 samples were obtained within the study period of 1 year i.e. May 2017 to April 2018. Out of these $519(519 / 532 \times 100=97.55 \%)$ samples were blood, 11 samples $(11 / 532 \times 100=2.06 \%)$ were from infected umbilical stump and remaining two were CSF $(2 / 532 \times 100=0.38 \%)$.

\section{Sample Preparation}

1. Blood- Blood was collected in the SNCU directly in the neonatal blood culture bottle, following strict aseptic precautions. The media supplied was brain, heart infusion broth. One in ten dilution volume for volume as blood: media was collected wherever possible. However, in case of some patients, enough blood could not be collected as the babies were very sick and dehydrated. The blood culture bottles were requisitioned from the department of Microbiology. The media was sterilized in the autoclave at $15 \mathrm{lb} / \mathrm{sq}$. inch pressure $121^{\circ} \mathrm{C}$ temperature before supply.

2. Pus from Umbilical Stump- Sterile disposable cotton swabs were used to collect pus from infected umbilical stump. Two such swabs were collected from each infected stump and immediately sent to the Microbiology department for processing.

3. CSF- CSF was collected in sterile test tube and sent to the Microbiology department.

\section{Methods of Examination}

Blood- Blood culture bottles were incubated at $37^{\circ} \mathrm{C}$. First examination was done after 24 hours of incubation. Subculture was done in blood agar plate and MacConkey's plate. The plates were incubated at $37^{\circ} \mathrm{C}$ overnight. Growth if any was examined and colony characters were noted. If not the plates were incubated for 48 hours and discarded if still no growth is obtained. On the event of no growth in first subculture, repeated subcultures were put up every alternate day for maximum of 7 days. Then the bottle was discarded. Smear was made from the colonies on subculture plates, stained by Gram-stain, examined under oil-immersion objective of the microscope and the findings were noted. A battery of biochemical tests were performed to identify the organism. After diagnosis of the isolate, antibiotic sensitivity 
test was performed by Kirby-Bauer technique as per CLSI guidelines.

Pus from Umbilical Stump- The culture was put up in blood agar and MacConkey's agar. Direct smear examination was also done, and culture was put up on Blood agar and MacConkey agar plates. Incubation was done for 24 to 48 hours. Isolate was diagnosed by a battery of biochemical reactions and antibiotic sensitivity was performed as above and result was tabulated.

CSF- The sample was examined macroscopically. Then microscopic examination was done as above. Centrifuged deposit was taken, smear prepared stained by Gram stain and examined for bacteria and pus cells. Culture was done and examined as above. Finally, antibiotic sensitivity test was done by Kirby-Bauer method following CLSI guidelines. Each sample obtained from the SNCU was examined as per departmental protocol-

1. Macroscopical Examination- Wherever applicable. Any abnormality seen in the specimen was noted.

2. Microscopically Examination- This was applicable for CSF and Pus. Smear was prepared from centrifuged deposit of CSF specimen and directly from swab obtained from Pus (in case of single swab smear was made after inoculation of culture plate).

The Smear was Stained by Gram Stain and Focussed under Oil Immersion Lens of the Microscope and the following Findings were noted-

a. Gram-positive/gram negative cocci or bacilli.

b. Presence of pus cells.

c. Round or oval yeast cells with or without budding/ pseudohyphae.

3. Culture-Inoculation was made on blood agar and MacConkey's agar media for primary culture. Blood was cultured in BHI broth and subcultured as per schedule in Blood agar and MacConkey agar plates. Special media were used depending on findings of smear examination. The twenty-four to forty-eight hours growth was thoroughly examined with reference to colony characters including size, shape, surface, margin, pigment production, haemolysis (in blood agar media), lactose fermentation (in MacConkey's media).

4. Biochemical Reactions- Biochemical reactions were performed according to departmental protocol and availability as shown in Table 1 . The tests to be performed were selected on the basis of Gram stain and culture findings.

5. Serological Test- Serological tests were performed based on availability of antisera and helped in confirmation of the isolates.

6. Antibiotic Sensitivity Test- Kirby-Bauer disc diffusion test was performed using Muller-Hinton media, following CLSI guidelines. For Gram-positive Cocci antibiotic discs used were cotrimoxazole, ceftriaxone, Clindamycin, doxycycline, gentamycin, levofloxacin, linezolid and vancomycin. For Gram-negative organisms, antibiotics used were piperacillin-tazobactam, imipenem, gentamicin, amikacin, ceftazidime,

\section{RESULTS}

Following macroscopical, microscopical, biochemical and serological tests wherever applicable, the organisms were identified as Klebsiella pneumoniae, Klebsiella oxytoca, Escherichia coli, Acinetobacter baumannii, Pseudomonas aeruginosa, Citrobacter koseri, Staphylococcus aureus (MR), Staphylococcus aureus (MS), LRSA MR cons, Enterococcus faecalis, Candida sp. A total of 532 samples were examined. Growth was obtained in 259 samples and 293 samples did not yield any growth. All the organisms were subjected to antibiotic sensitivity test by Kirby-Bauer disc diffusion test. Antibiotic discs used were subject to CLSI guidelines and availability in the departmental bacteriology laboratory. MRSA and MR Concs were assessed phenotypically by Cefoxitin resistance.

\begin{tabular}{|c|c|}
\hline $\begin{array}{c}\text { Smear Showing } \\
\text { Gram Positive Cocci. }\end{array}$ & $\begin{array}{c}\text { Smear Showing } \\
\text { Gram Negative Bacilli }\end{array}$ \\
\hline $\begin{array}{c}\text { (1) Catalase test } \\
\text { (2) Oxidase test } \\
\text { (3) Coagulase test } \\
\text { (a) Slide coagulase test } \\
\text { (b) Tube coagulase test } \\
\text { (4) Urease test } \\
\text { (5) VP test } \\
\text { (7) PYR test } \\
\text { (8) ODC test } \\
\text { (6) Glucose and other sugar fermentation } \\
\text { Novobiocin } \\
\text { (9) Sensitivity to polymyin B, } \\
\text { (10) Only for suspected enterococcus, } \\
\text { Arabinose fermentation and growth in } \\
\text { bile aesculin medium }\end{array}$ & $\begin{array}{l}\text { (1) Catalase test } \\
\text { (2) Oxidase test } \\
\text { (3) Hanging drop preparation to see } \\
\text { motility } \\
\text { (4) Nitrate reduction test } \\
\text { (5) Indole test } \\
\text { (6) MR test } \\
\text { (7) VP test } \\
\text { (8) Urease test } \\
\text { (9) Citrate Utilization test } \\
\text { (10) Growth in TSI medium } \\
\text { (11) Sugar fermentation test } \\
\text { (12) ODC test } \\
\text { (13) LDC test } \\
\text { (14) ADH test } \\
\text { (15) For NLF organism } \\
\text { The above test+ } \beta \text {-galactosidase test } \\
\text { (ONPG and PPA test) }\end{array}$ \\
\hline
\end{tabular}

\begin{tabular}{|c|l|l|c|}
\hline Total No. of Samples & No. & $\mathbf{\%}$ \\
\hline 532 & Total number of samples yielding growth & 259 & $48 \%$ \\
\hline 532 & Total number of samples not yield growth & 293 & $52 \%$ \\
\hline 532 & Total number of samples yielding growth & 259 & $48 \%$ \\
\hline 532 & Total number of samples not yield growth & 293 & $52 \%$ \\
\hline Table 2. Percentage of Positive and Negative Samples \\
\hline \multicolumn{4}{|l}{} \\
\hline
\end{tabular}

\begin{tabular}{|c|c|c|c|}
\hline Blood & Pus & CSF & Total \\
\hline Klpn & & & \\
\hline 100 & 11 & 0 & 111 \\
\hline Klox & & & \\
\hline 0 & 1 & 0 & 1 \\
\hline E.coli & & & \\
\hline 17 & 8 & 1 & 26 \\
\hline Aci & & & \\
\hline 9 & 4 & 0 & 13 \\
\hline Pseudo & & & \\
\hline 8 & 0 & 0 & 8 \\
\hline $\begin{array}{c}\text { Citro } \\
9\end{array}$ & 1 & 0 & 10 \\
\hline MRSA & & & 10 \\
\hline & 1 & 0 & 23 \\
\hline 28 & 20 & 0 & 48 \\
\hline LRSA & & & \\
\hline 1 & 0 & 0 & 1 \\
\hline Cons & & & \\
\hline $\begin{array}{c}4 \\
\text { MR Cons }\end{array}$ & 0 & 0 & 4 \\
\hline 2 & 0 & 0 & 2 \\
\hline Ent faec & & & \\
\hline $\begin{array}{c}0 \\
\text { Candida }\end{array}$ & 3 & 0 & 3 \\
\hline 8 & 0 & 1 & 9 \\
\hline 208 & 49 & 2 & 259 \\
\hline
\end{tabular}




\section{Gram Positive Bacteria}

$100 \%$ of the gram-positive bacteria isolated were sensitive to vancomycin. In case of Linezolid only one Staph aureus isolate was resistant. Levofloxacin and Gentamycin showed $60 \%$ sensitivity followed closely by doxycycline $-50 \%$ sensitivity. Cotrimoxazole, ceftriaxone and clindamycin showed $40 \%$ sensitivity and the bacteria were least sensitive to amoxycillin-clavulanic acid $-20 \%$ sensitivity.

\section{Gram Negative Bacteria}

In case of Gram-negative bacilli all the isolates were sensitive to colistin (colistin was used at this time). The isolates showed $80 \%$ sensitivity to imipenem, $70 \%$ to piperacillintazobactam and $40 \%$ to cefepime. Gentamicin and amikacin were effective in $35 \%$ cases ceftazidime in 30\% whereas Cefotaxime and Cotrimoxazole and doxycycline were effective in $20 \%$ isolates each.

\section{DISCUSSION}

Several previous studies involving SNCU infection were compared with the results of the present study. A lot of similarities as well as differences were noted. As per the results of this study the total number of clinical cases examined were 532. Out of these 259 were growth positive which comes to $48 \% .52 \%$ of the samples did not produce any growth. The reasons for this may be diverse, such as, no infection present, presence of a fastidious organism which requires specific growth factors, presence of slow growers etc. On comparing this number with other studies, we found that the numbers were extremely varied nationally and internationally. In a study by Basavaraj et al (2013) in Bijapur Karnataka. ${ }^{1}$

Karnataka the percentage of culture positive cases is $19.2 \%$. Leela et al (2016) in a study conducted at Chennai, 2 India gave a positivity rate of $35.77 \%$. In 2015 AIIMS, Jodhpur reported $22 \%$ of culture positive cases. Tiwari et al (2013) from Bangalore ${ }^{3}$ reported $25 \%$ culture positivity. A study in Port Harcourt by West et al in $2012^{4}$ reports $41.6 \%$ positivity. Our study shows the growth of a variety of Gram-positive and Gram-negative organisms. Gram negative organisms by far exceeded the number of gram-positive organisms. The percentage of the former was $65.25 \%$ and latter was $31.27 \%$. In our study 9 cases of Candida sp. were isolated from blood$3.47 \%$. This value has been corroborated by many other studies in India and abroad. Dr. Karthick et al in 2016 BMCRI Bangalore $^{5}$ showed a percentage value of $58.5 \%$ Gram negative and 28.61. Gram-positive and $8.3 \%$ Candida sp. of which 95.4\% were non albicans Candida. Pal et al (2011) from Jodhpur AIIMS ${ }^{6}$ reported $61.25 \%$ Gram negative and $38.75 \%$ Gram-positive isolates. Leela et al from Chennai ${ }^{2}$ also had a large excess of Gram-negative isolates. The Port Harcourt study ${ }^{4}$ showed $75.1 \%$ Gram negative isolates and 24.The Gram-positive. Basavaraj from Bijapur Karnataka ${ }^{1}$ reported 55.7\% Gram negative and $44.3 \%$ Gram-positive organisms.

The commonest organism isolated in our study was Klebsiella pneumoniae (42.85\%) followed by Staphylococcus aureus $(18.53 \%)$. This is followed by E. coli $(10.03 \%)$, MRSA
(8.88\%), Acinetobacter baumannii (5.01\%), Citrobacter koseri (3.86\%), Pseudomonas aeruginosa (3.08\%) and few isolates of Staphylococcus epidermidis, Enterococcus faecalis, LRSA and Candida sp. Klebsiella pneumoniae were the predominant organism isolated in all the above studies including ours except in AIIMS Jodhpur were Staphylococcus aureus were the predominant species isolated. Candida sp. were mentioned in our study and in the study of Karthick et al. from Bangalore ${ }^{5}$ Cons has been found following Klebsiella pneumoniae by Leela $^{2}$ et al. and Basavaraj ${ }^{1}$ et al. Remaining workers showed Staphylococcus aureus isolate following Klebsiella pneumoniae. Coming to the antibiotic sensitivity pattern of the organism in our study, Gram negative organisms were susceptible to imipenem in $80 \%$ of cases followed by piperacillin tazobactam (70\%).

Cefepime (40\%), Gentamicin (35\%) and amikacin (35\%), Ceftazidime $(30 \%)$, Cefotaxime (25\%), Cotrimoxazole and Doxycycline (20\%) each. Gram-positive organisms were 100 $\%$ sensitive to vancomycin. Only one isolate was LRSA. Levofloxacin, Gentamicin sensitivity was seen in $60 \%$ isolates. $50 \%$ sensitivity was seen in doxycycline, $40 \%$ in Clindamycin, and Ceftriaxone. Cotrimoxazole and amoxiclav showed only $20 \%$ sensitivity. The drug sensitivity pattern or AST pattern obtained in our study is corroborated by findings of the previously mentioned studies with a few exceptions for example Pal in Jodhpur6 showed vancomycin sensitivity in $40 \%$ whereas in our study all the Gram-positive isolates were sensitive to vancomycin. Linezolid sensitivity has not been mentioned in these studies except Basavaraj et al.

\section{CONCLUSIONS}

One important point that we have noted is that certain antibiotics like cotrimoxazole which had gone out of circulation for some time have regained their sensitivity in recent times. Resistance patterns of antibiotics are changing and when they are being removed from regular use, they are regaining their sensitivity in some cases. This technique could be used as a rule in future to recirculate good time-tested drugs. While doing the groundwork for these studies it was found that the organists along with their antibiotic sensitivity pattern were constantly changing. As a result, such studies should be an ongoing process to be conducted methodically at regular intervals particularly in a developing country like India where infection is acquired easily. Importance should also be given to strict and regular infection control methods and surveillance. According to drug resistance studies, we can clearly see that narrow spectrum antibiotics like penicillin are gradually becoming less useful. Newer antibiotics like later generation cephalosporins, carbapenems, fluorquinolones and oxazolidinone groups of antibiotics are being increasingly used. Thus, the utility of such a study lies in formulation of a logical and calculated antibiotic policy in a health set up. This will go a long way in helping paediatricians to begin empirical treatment early in case of neonatal sepsis cases and save the life of thousands. 


\section{REFERENCES}

[1] Jyothi P, Basavaraj MC, Basavaraj PV. Bacteriological profile of neonatal septicemia and antibiotic susceptibility pattern of the isolates. J Nat Sc Biol Med 2013;4(2):306-9.

[2] Leela KV, Babu RN, Prasad VM, et al. Study of bacterial profile in neonatal sepsis and their antibiotic sensitivity pattern in a tertiary care hospital. Int J Curr Microbiol App Sci 2016;5(6):511-21.

[3] Tiwari DK, Golia S, Sangeetha KT, et al. A study on the bacteriological profile and antibiogram of bacteremia in children below 10 years in a tertiary care hospital in Bangalore, India. J Clin Disgn Res 2013;7(12):2732-5.
[4] West BA, Peterside 0. Sensitivity pattern among bacterial isolates in neonatal septicaemia in Port Harcourt. Ann Clin Microbiol Antimicrob 2012;11:7.

[5] Karthik R, Nagarathnamma T. Microbial profile and antibiogram of neonatal septicaemia at a tertiary care hospital in Bangalore. Int J Curr Res 2016;8(4):29513-7.

[6] Tsering DC, Chanchal L, Pal R, et al. Bacteriological profile of septicaemia and the risk factors in neonates and infants in Sikkim. J Glob Infect Dis 2011;3(1):42-5. 